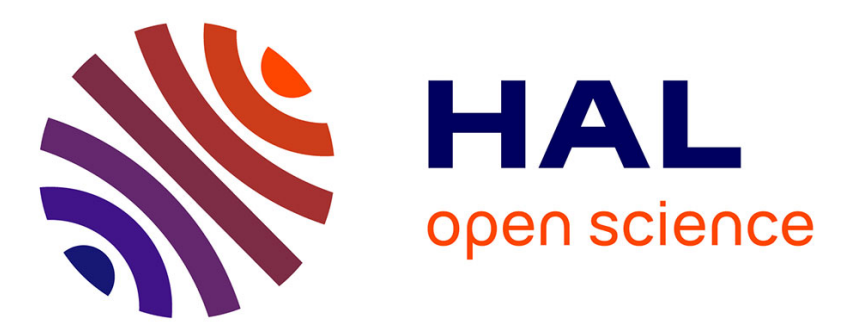

\title{
Influence of the growth conditions on the defect density of single-walled carbon nanotubes
}

Matthieu Picher, Hugo Navas, Raul Arenal, Etienne Quesnel, Eric Anglaret, Vincent Jourdain

\section{- To cite this version:}

Matthieu Picher, Hugo Navas, Raul Arenal, Etienne Quesnel, Eric Anglaret, et al.. Influence of the growth conditions on the defect density of single-walled carbon nanotubes. Carbon, 2012, 50, pp.2407. 10.1016/j.carbon.2012.01.055 . hal-00802066

\section{HAL Id: hal-00802066 https://hal.science/hal-00802066}

Submitted on 21 Mar 2013

HAL is a multi-disciplinary open access archive for the deposit and dissemination of scientific research documents, whether they are published or not. The documents may come from teaching and research institutions in France or abroad, or from public or private research centers.
L'archive ouverte pluridisciplinaire HAL, est destinée au dépôt et à la diffusion de documents scientifiques de niveau recherche, publiés ou non, émanant des établissements d'enseignement et de recherche français ou étrangers, des laboratoires publics ou privés. 


\section{Influence of the growth conditions on the defect density}

\section{of single-walled carbon nanotubes}

Matthieu Picher ${ }^{1,2}$, Hugo Navas ${ }^{1,2}$, Raul Arenal $^{3,4}$,Etienne Quesnel ${ }^{5}$, Eric Anglaret ${ }^{1,2}$, Vincent Jourdain $^{1,2, *}$

${ }^{1}$ Université Montpellier 2, Laboratoire Charles Coulomb UMR 5221, F-34095, Montpellier, France

${ }^{2}$ CNRS, Laboratoire Charles Coulomb UMR 5221, F-34095, Montpellier, France

${ }^{3}$ Laboratoire deetude des microstructures, UMR 104 CNRS-ONERA, 29 av. de la Division Leclerc, 92322 Châtillon, France

${ }^{4}$ Laboratorio de microscopias avanzadas (LMA), Instituto de Nanociencia de Aragon (INA), U. Zaragoza, C/ Mariano Esquillor s/n, 50018 Zaragoza, Spain.

${ }^{5}$ CEA-LITEN, 17 rue des martyrs, 38054 Grenoble cedex 9, France.

*Corresponding author. E-mail address: vincent.jourdain@univ-montp2.fr 


\section{ABSTRACT}

The influence of the temperature and precursor pressure on the defect density of single-walled carbon nanotubes (SWCNTs) grown by catalytic chemical vapor deposition was studied for several catalystprecursor couples. The SWCNT defect density was assessed by studying the Raman D band. In situ Raman monitoring was used to determine experimental conditions allowing the preparation of samples free of pyrolytic carbon and not altered by air exposure. The most striking feature is that the Arrhenius plots of the $\mathrm{I}_{\mathrm{G}} / \mathrm{I}_{\mathrm{D}}$ ratio systematically display a convex shape, i.e. the apparent activation energy decreases with increasing temperature. From HRTEM observations and oxidation experiments, this evolution of the D band features is ascribed to the catalytic growth of long SWCNTs with few defects at high temperature and of short and defective SWCNTs and carbon structures at low temperature. The convex Arrhenius behavior is well accounted by two kinetic models: i) a model considering a change of intermediate states as a function of the temperature (for instance due to a phase transition of the catalyst particle or a change of intermediate carbon species), and ii) a model considering a high-temperature process of defect creation (for instance by reaction with reactive gas species). 


\section{Introduction}

Catalytic chemical vapor deposition (CCVD) is the most widely used technique for growing singlewalled carbon nanotubes (SWCNTs). It is also the most promising one in terms of upscaling and structural control. Despite that, it is commonly observed that as-grown SWCNTs contain a certain amount of defects. Controlling the defect density of SWCNTs (and graphene-type materials in general) is a key issue for the control of their properties. Defects severely affect the electronic and optical properties of SWCNTs [1,2], and are notably responsible for a drastic drop of conductivity [1]. By contrast, one can take advantage of defects to tune the electronic properties of SWCNTs [3]. Defects can also act as preferential sites of functionnalization and the density of defects is therefore a key parameter for controlling the density of grafted functions. Contrary to the dependence of SWCNT properties on defects, little is known about the elementary processes of defect creation during the nanotube growth and their potential role in the growth mechanism. Theoretically, the integration of a single defect may dramatically influence the growth of a one-dimensional crystal such as SWCNT. From Euler s rule, the integration of a single pentagonal or heptagonal ring will convert a growing tube into a cone. Therefore, the integration of a single defect may lead to the termination of the tube growth. Alternatively, the integration of a few defects may create an elbow connection and change the chirality of the growing tube [4]. In the simulations of nanotube growth, the formation of defective structures at the nanotube rim (e.g. chains, non-hexagonal rings $\square$ ) is commonly observed [5,6]. However, very long SWCNTs can be experimentally grown at growth rates as high as several tens of microns per second [7]. This suggests either a remarkably efficient mechanism preventing the formation of defects or a remarkably efficient mechanism of defect annealing.

Raman spectroscopy is widely used to study defects in $\mathrm{sp}^{2}$ carbon-based materials $[8,9]$, most commonly through the measurement of the intensity ratio of the defect-induced D band to the graphitic G band $[10,11]$. The $\mathrm{D}$ band is theoretically non-active in Raman scattering but is activated in the presence of defects through a double resonance process involving the elastic scattering of electrons by defects 
$[12,13]$. The $I_{G} / I_{D}$ ratio has been widely used to estimate the density of defects in nanotubes and graphene [12-16]. When the average distance between defects $\left(L_{D}\right)$ in graphene is larger than a few nm, the ratio $I_{G} / I_{D}$ is inversely proportional to the surface density of defects $(\sigma)$ following the relation:

$$
\frac{I_{G}}{I_{D}}=K \sigma^{-1}
$$

where $\mathrm{K}$ is a parameter that depends on the laser energy [17]. When $L_{D}$ reaches a few nanometers, the defects start to coalesce causing the $\mathrm{D}$ band to broaden and $\mathrm{I}_{\mathrm{G}} / \mathrm{I}_{\mathrm{D}}$ to decrease with increasing defect density. The $\mathrm{D}$ band is dispersive, i.e. its frequency $\omega_{\mathrm{D}}$ changes with the excitation laser energy, $\mathrm{E}_{\text {laser, }}$ with $\Delta \omega_{\mathrm{D}} / \Delta \mathrm{E}_{\text {laser }} \sim 50 \mathrm{~cm}^{-1} / \mathrm{eV}$, a value that was shown to be almost independent of the type of $\mathrm{sp}^{2}$ carbon material [9]. The D-band profile is also dependent on the type and structure of the $\mathrm{sp}^{2}$ carbon material. For instance, Souza Filho et al. [18] showed that for SWCNTs, the frequency of the D band is dependent on the nanotube diameter following the general trend $\omega_{\mathrm{D}}=1354.8-16.5 / \mathrm{d}$ for $\mathrm{E}_{\text {laser }}=2.41 \mathrm{eV}$. For a SWCNT sample, this would translate into a D-band downshift of $10-20 \mathrm{~cm}^{-1}$ (depending on the diameter distribution) relatively to graphene and other flat $\mathrm{sp}^{2}$ carbon materials. For this reason, SWCNT display the lowest $\omega_{\mathrm{D}}$ of reported $\mathrm{sp}^{2}$ carbon materials (see Table S5 in Supplementary Information). The linewidth of the D band significantly depends on the type and structure of the $\mathrm{sp}^{2}$ carbon material. Owing to quantum confinement effects, an individual SWCNT usually displays a narrow D-band: the typical linewidth is $20 \mathrm{~cm}^{-1}$ [19] and can be as narrow as $7 \mathrm{~cm}^{-1}$ [20]. It follows that the D-band of a SWCNT sample is usually broader than the D-band of an individual SWCNT and that the linewidth depends on the diameter distribution of the sample. Single-layer graphene with a small amount of defects also displays a quite narrow D-band with a linewidth around $20 \mathrm{~cm}^{-1}$ which broadens for $\mathrm{L}_{\mathrm{D}}<5 \mathrm{~nm}$ [17]. Graphitic $\mathrm{sp}^{2}$ carbon materials (graphite polyhedral crystal, graphite whiskers) can also display D-band linewidth around $20 \mathrm{~cm}^{-1}$ [21] while less ordered materials (CVD multi-walled nanotubes [22], turbostratic carbon [21], short stacked graphene patches [23], polycrystalline graphite [24] amorphous carbon [25] $\square$ ) display a broad $\mathrm{D}$ band with linewidths ranging from $45 \mathrm{~cm}^{-1}$ to $100 \mathrm{~cm}^{-1}$. 
Usually, the D band of a SWCNT sample is considered to be the sum of two contributions: one related to defects in the SWCNTs and another one related to defective carbon by-products. However, the exact nature of the defective by-products (pyrolitic amorphous carbon, catalytically-grown defective structures, carbon filaments, carbon shells $\square$ ) is not always known. Arepalli et al. reported that after a soft air treatment, the D-band linewidth of a SWCNT HiPCO sample decreased from $\sim 60$ to $30 \mathrm{~cm}^{-1}$ without modifying the nanotube diameter distribution [26]. Such observations support that both SWCNT and more defective carbon forms contribute to the D band of standard SWCNT samples. Even though the Raman signal of SWCNT is enhanced by several orders of magnitude by resonance effect, the contribution of highly defective carbon materials to the D band of a SWCNT sample could be non negligible for a sufficiently large amount of defective by-products.

A few studies have been devoted to the growth parameter dependence of the $\mathrm{I}_{\mathrm{G}} / \mathrm{I}_{\mathrm{D}}$ ratio. Vinten et al. reported ex situ Raman measurements showing that the $\mathrm{I}_{\mathrm{G}} / \mathrm{I}_{\mathrm{D}}$ ratio increases with increasing growth temperature until an optimal temperature, then stabilizes and finally decreases at higher temperatures [14]. The temperature- and time-evolution of the $\mathrm{I}_{\mathrm{G}} / \mathrm{I}_{\mathrm{D}}$ ratio were also studied by Kwok et al. [15] by ex situ Raman measurements. In agreement with Vinten et al., they reported that the $\mathrm{I}_{\mathrm{G}} / \mathrm{I}_{\mathrm{D}}$ ratio increased, stabilized and then decreased with increasing growth temperatures. In addition, they showed that the decrease of the $I_{G} / I_{D}$ ratio observed at high temperature was amplified when increasing the growth duration and attributed the phenomenon to the co-deposition of amorphous carbon at high temperature during SWCNT growth. By combining in situ thermal analysis and TEM observations, Feng et al. $[14,15,27]$ confirmed that the co-deposition of defective carbon impurities was promoted at high growth temperatures and high precursor pressures. These studies essentially addressed the contamination of SWCNT samples by pyrolytic defective by-products. However, the processes controlling the type and density of defects integrated in the SWCNTs during their growth are still ill-understood.

In a previous report [28], we observed a correlation between the characteristic time of growth termination and the defect density of the SWCNTs. We also observed that the $I_{G} / I_{D}$ ratio followed an 
Arrhenius-type regime as a function of the growth temperature. In the present article, we report a more systematic investigation of the temperature and precursor pressure dependence of the defect density during SWCNT growth for several catalyst-precursor systems by Raman spectroscopy. In situ monitoring by Raman spectroscopy allowed us to identify two phenomena masking the intrinsic Raman signatures of as-grown SWCNTs and to choose experimental conditions to prevent them. In these conditions, we observed that the temperature dependence of the $I_{G} / I_{D}$ ratio systematically displays an unusual convex Arrhenius plot [29], i.e. two different Arrhenius regimes marked by lower activation energy at high temperature. Analyses of the D-band features coupled with HRTEM observations and controlled oxidation experiments were performed to identify the carbon nanostructures grown in each temperature domain. We find that long and few-defects SWCNT dominate the samples grown at high temperatures and low precursor pressure while low temperatures and high precursor pressures promote the catalytic growth of very short and defective SWCNTs. These experimental results are well accounted by two different kinetic models. Finally, we discuss the possible microscopic origins and the implications of these findings for the growth mechanism of SWCNTs.

\section{Materials and Methods}

Thin layers $(5 \AA)$ of nickel or cobalt were deposited by evaporation or ion beam sputtering onto $5 \times 5 \mathrm{~mm}$ (100) silicon substrates with a $100 \mathrm{~nm}$ thermal oxide layer. CCVD experiments were achieved in a coldwall reactor cell (Linkam TS-1500 stage) equipped with a resistive heating crucible, an integrated thermocouple and a silica window allowing optical measurements. Four different carbon precursors (ethanol, methane, ethylene and acetylene) were investigated. Ethanol vapor was supplied into the reactor by bubbling argon (Air liquid class 2, 99.996\%) through a thermostated ethanol flask (Fluka, 99.8\%). An additional diluting argon line was used to precisely adjust the partial pressure of the carbon precursor before introduction into the reactor cell. To prevent catalyst coarsening, each sample was pretreated in oxygen from room temperature to $700^{\circ} \mathrm{C}$ at a rate of $50^{\circ} \mathrm{C} / \mathrm{min}$ [30]. The cell was then purged 
with $1400 \mathrm{sccm}$ of argon for 10 min while reaching the desired synthesis temperature. The growth was monitored in situ by Raman measurements with a micro-Raman spectrometer (Jobin Yvon T-64000, simple grating configuration). When no more evolution of the Raman spectra was observed, the cell was cooled to room temperature at $100^{\circ} \mathrm{C} / \mathrm{min}$. Typical synthesis times ranged from 1 to $30 \mathrm{~min}$ depending on the growth conditions. Before opening to air, Raman spectra of each sample were acquired at room temperature under argon flow. Raman measurements were performed at either $532 \mathrm{~nm}$ or $647 \mathrm{~nm}$ with a $\times 50$ objective lens. For HRTEM analysis, the samples were transferred to TEM grids by scraping the $\mathrm{SiO}_{2} / \mathrm{Si}$ substrates. HRTEM observations were carried out using a Zeiss Libra $200 \mathrm{FE}$ (working at $200 \mathrm{kV}$ ) and two FEI Tecnai: a F20 (working at 200kV) and F30 (working at $80 \mathrm{kV}$ ).

\section{Experimental results}

A first observation resulting from in situ Raman monitoring is that the intrinsic $\mathrm{D}$ band and $\mathrm{I}_{\mathrm{G}} / \mathrm{I}_{\mathrm{D}}$ ratio of as-grown SWCNTs can be masked by extrinsic effects. Figures 1a-b shows two typical evolutions of the integrated intensity of the $\mathrm{G}$ and $\mathrm{D}$ bands as a function of the synthesis time. Most frequently, both intensities increase continuously and finally reach a plateau after a time ranging from several seconds to tens of minutes depending on the growth conditions (figure 1a). The same behavior was reported by $\mathrm{Li}^{-}$ Pook-Than et al. [31]. Consequently, the $\mathrm{I}_{\mathrm{G}} / \mathrm{I}_{\mathrm{D}}$ ratio reaches a stable value at the end of the growth. In this case, the final value only depends on the growth conditions and does not depend on the growth duration. Noteworthy exceptions occur for high temperature and high precursor pressure growths, as shown in figure $1 \mathrm{~b}$. In these growth conditions, the $\mathrm{G}$ band goes through a maximum then slowly decreases while the $\mathrm{D}$ band keeps increasing slowly. Consequently, the $\mathrm{I}_{\mathrm{G}} / \mathrm{I}_{\mathrm{D}}$ ratio continuously decreases with synthesis time. In these conditions, the value of $\mathrm{I}_{\mathrm{G}} / \mathrm{I}_{\mathrm{D}}$ depends on the time at which the synthesis is stopped. The corresponding D band is very broad with a full width at half maximum (FWHM) of 100$120 \mathrm{~cm}^{-1}$. It is worth noting that this behavior is accompanied by a progressive and continuous decrease of the Raman peaks of the silicon substrate. This behavior is in close agreement with the co-deposition of 
amorphous carbon at high growth temperature observed by several groups and characterized by a decrease of $I_{G} / I_{D}$ and a broadening of the $D$ band at high temperature [15,16,32]. During our investigations, we only observed this behavior in the high temperature-high precursor pressure corner of the parameter range, which may be explained by the use of a cold-wall reactor. Hereafter, we only report on growth conditions that prevent this contamination by pyrolytic amorphous carbon (i.e. no increase and broadening of the $\mathrm{D}$ band with increasing temperature, no decrease of the $\mathrm{G}$ band and silicon peaks with increasing time). Under these conditions, the Raman spectra are characteristic of the carbon structures that have been catalytically grown and are not modified by the co-deposition of amorphous pyrolytic carbon. All the data presented in the following therefore correspond to the kinetic behavior presented in Figure 1a.
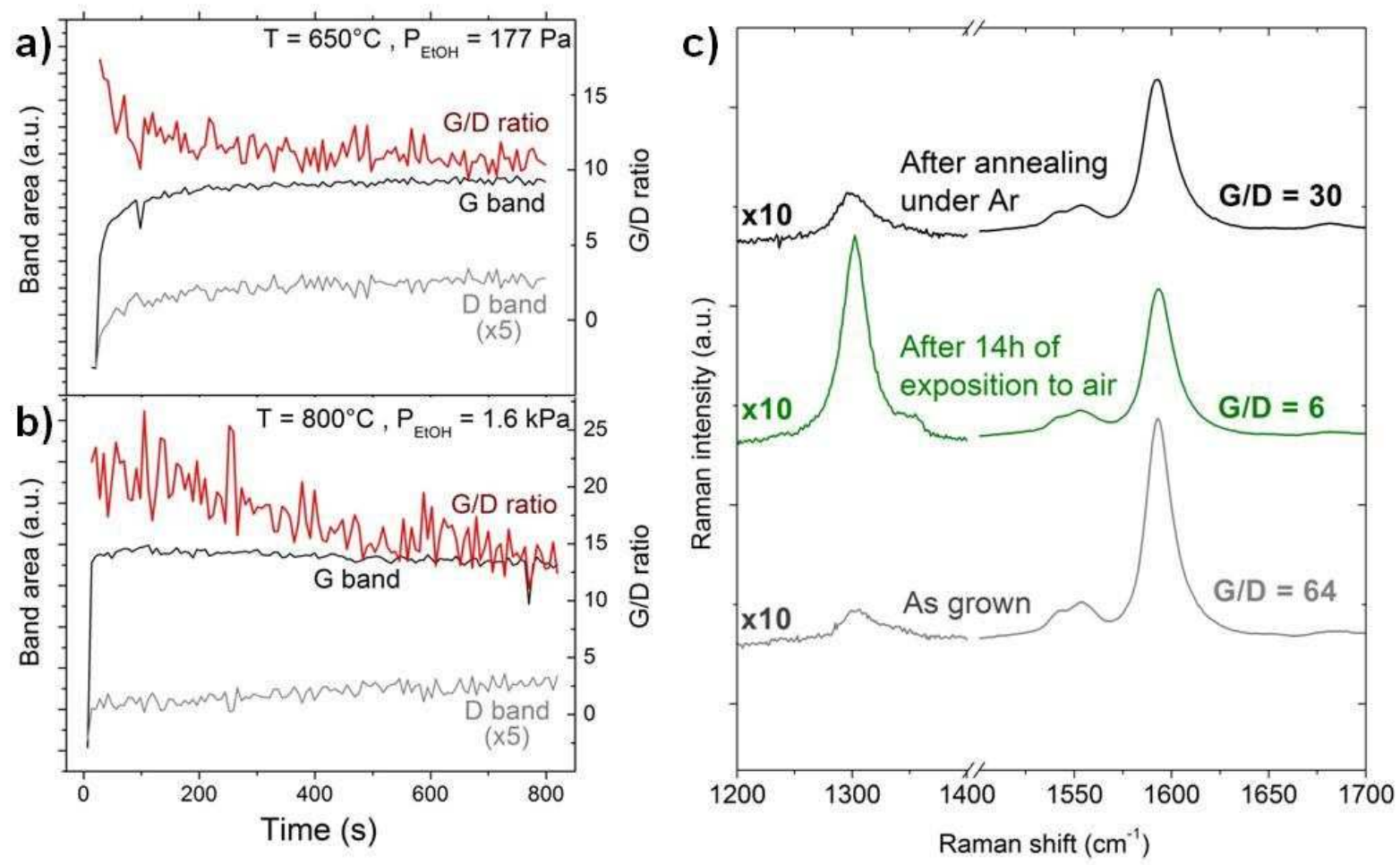

Figure 1. Time evolutions of the intensities of $G, D$ and of the $G / D$ ratio (nickel-ethanol couple): a) standard behavior, b) high temperature-high pressure behavior. c) Evolution of the Raman spectra of SWCNT samples: as-grown (grey line), after $14 \mathrm{~h}$ of exposition to air (green line) and after annealing 
under Ar at $650^{\circ} \mathrm{C}$ (black line). The laser excitation line is $647 \mathrm{~nm}$. All D bands are multiplied by a factor 10 .

We also observed that the $\mathrm{D}$ band and the $\mathrm{I}_{\mathrm{G}} / \mathrm{I}_{\mathrm{D}}$ ratio of some samples were significantly modified after a few hours of exposition to air (Figure 1c). Just after opening the cell to air, no modification of the D band could be observed compared to the spectra measured under argon inside the cell. However, after several hours of air exposure, the D band could be increased by as much as a factor 8 (fig. 1c) and the $\mathrm{I}_{\mathrm{G}} / \mathrm{I}_{\mathrm{D}}$ ratio decreased by a factor 10 . The $\mathrm{I}_{\mathrm{G}} / \mathrm{I}_{\mathrm{D}}$ ratio can be significantly (but not completely) recovered by annealing the samples under argon (fig. 1c). This phenomenon cannot be explained by a simple laserinduced degradation of the SWCNTs under air because it only appears after several hours of air exposure and was then observed all over the sample even at the lowest laser power. We attribute this behavior to the slow adsorption of molecules present in the air. A similar phenomenon was observed by Shim et al. when exposing SWCNTs to pure oxygen [33]. For the present study, it is important to prevent this phenomenon in order to measure the intrinsic $I_{G} / I_{D}$ ratio of as-grown SWCNT. For this reason, all the post-growth Raman data presented hereafter were recorded under argon just after cooling the sample to room temperature and before opening the cell to air.

Figure $2 \mathrm{a}$ shows the evolution of the post-growth Raman spectra as a function of the synthesis temperature for the cobalt-acetylene couple. It is observed that the $\mathrm{I}_{\mathrm{G}} / \mathrm{I}_{\mathrm{D}}$ ratio increases with increasing temperature (figure 2b). This is in agreement with the reports of Vinten et al. [12] and Kwok et al. [13] except that there is no decrease of the $\mathrm{I}_{\mathrm{G}} / \mathrm{I}_{\mathrm{D}}$ ratio at high temperature since we focused here on growth conditions free of pyrolitic carbon deposition. Figure 2a shows that the D band is the sum of two distinct components: a narrow $\mathrm{D}_{1}$ peak at $\omega_{\mathrm{D} 1} \sim 1300 \mathrm{~cm}^{-1}$ and a broader $\mathrm{D}_{2}$ band at $\omega_{\mathrm{D} 2} \sim 1322 \mathrm{~cm}^{-1}$ (for a laser excitation line of $1.92 \mathrm{eV}$ ). The two components were fitted using Lorentzian features. Figures $2 \mathrm{c}$ and $2 \mathrm{~d}$ show the evolution of the position and FWHM of each component, respectively, as a function of the reciprocal growth temperature. The positions of the components are essentially independent of the 
growth temperature. The FWHM of $\mathrm{D}_{1}$ is about $30 \mathrm{~cm}^{-1}$ and displays a weak variation with increasing temperature. The FWHM of $\mathrm{D}_{2}$ is $\sim 75 \mathrm{~cm}^{-1}$ at the lowest temperatures $\left(650-675^{\circ} \mathrm{C}\right)$ and more markedly decreases with increasing temperature. Interestingly, $\mathrm{D}_{1}$ dominates the $\mathrm{D}$ band at high temperature while $\mathrm{D}_{2}$ dominates the $\mathrm{D}$ band at low temperatures (fig. $2 \mathrm{~b}$ ). As a consequence, the overall position of the $\mathrm{D}$ band progressively downshifts from $\omega_{\mathrm{D} 2}$ to $\omega_{\mathrm{D} 1}$ with increasing temperature. Figure $\mathrm{S} 1$ in Supplementary Information presents the same behavior at a different laser energy (cobalt-acetylene samples characterized at $\mathrm{E}_{\text {laser }}=2.33 \mathrm{eV}$ ). All the catalyst-precursor couples that we studied (nickel-acetylene, nickel-ethylene, nickel-methane, cobalt-ethanol, cobalt acetylene) displayed this overall downshift and narrowing of the D band with increasing temperature (see figure S2). Remarkably, the two components of the D band are best resolved for samples with the largest proportion of small-diameter SWCNTs as deduced from the radial breathing mode (RBM) profiles (figure $2 a$ ).
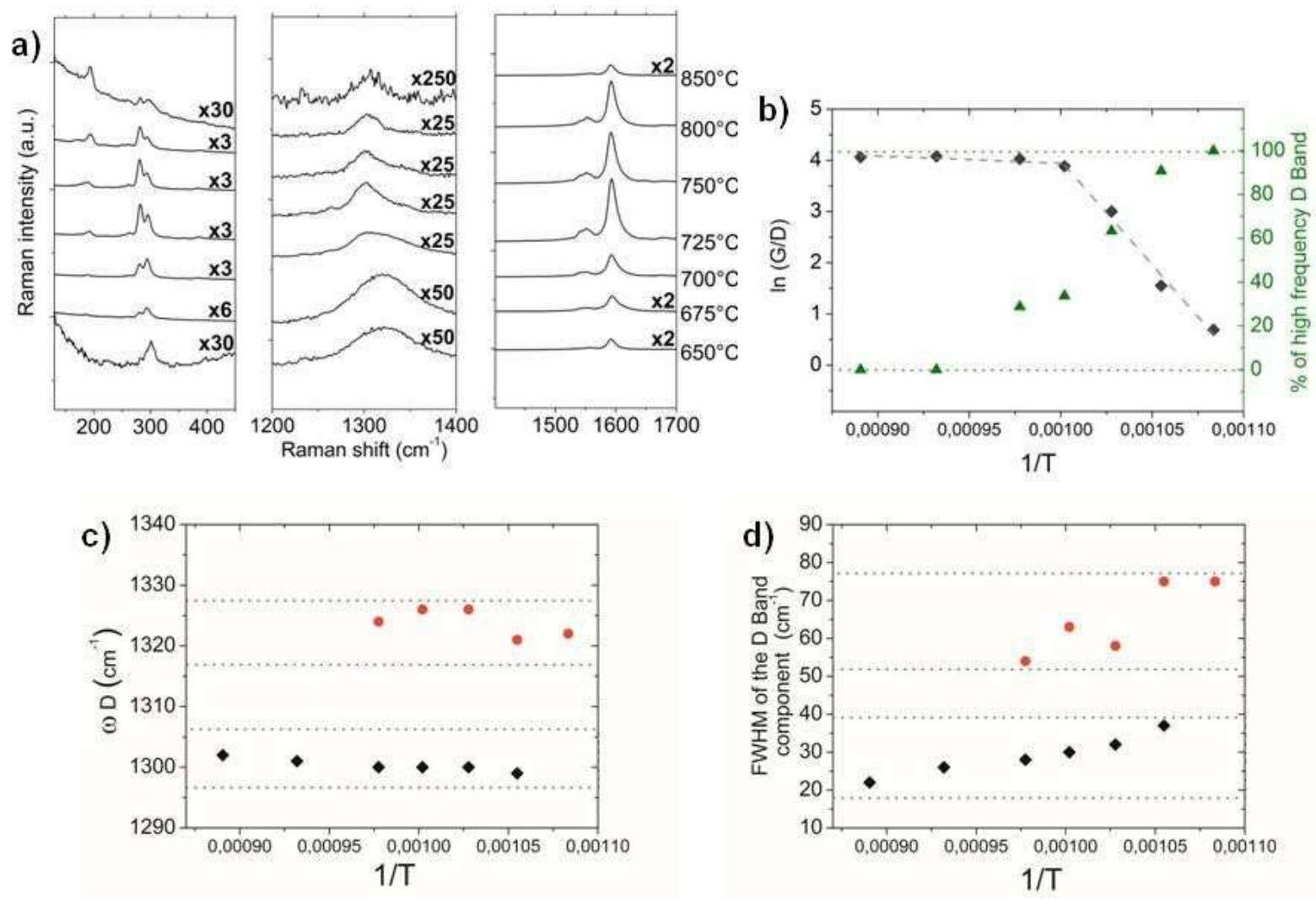

Figure 2. SWCNT samples grown with the cobalt-acetylene couple: a) room-temperature Raman spectra as a function of the growth temperature, b) G/D ratio (diamonds) and proportion of the high 
frequency component ( $\left.D_{2}\right)$ in the D band (triangles), c) positions and d) FWHM of the two components of the D band against inverse temperature. The laser excitation line is $647 \mathrm{~nm}$. The dashed lines in fig. $2 b$ are fitted curves based on models $A$ and $B$.

Figure 3 shows Raman results for samples grown with another system (ethanol precursor and nickel catalyst) for which a systematic study of the influence of the precursor pressure and growth temperature was performed. A general observation is that the $\mathrm{I}_{\mathrm{G}} / \mathrm{I}_{\mathrm{D}}$ ratio decreases with decreasing growth temperature and increasing precursor pressure (figure 3a). Figures $3 \mathrm{~b}$ and $3 \mathrm{c}$ show typical Raman spectra of samples prepared at high and low temperatures, respectively. At high temperatures, the spectra are typical of SWCNT. In the range of RBM $\left(150-350 \mathrm{~cm}^{-1}\right)$, the whole bunch is quite intense and each RBM component is well resolved. In the range of tangential modes (TM), the G band displays two wellresolved components (the so-called $\mathrm{G}^{-}$and $\mathrm{G}^{+}$components [12]) and is much stronger than the $\mathrm{D}$ band. The FWHM of the D band is about $40 \mathrm{~cm}^{-1}$. By contrast, at low temperatures (fig. 3c), the G/D ratio is much lower, the RBM are weaker and the D band is broader (FWHM of about $70 \mathrm{~cm}^{-1}$ ). If one further decreases the temperature down to $500^{\circ} \mathrm{C}$ (see figure $\mathrm{S} 3$ in Supp. Info.), the RBM become extremely weak and ill-resolved, the D band broadens even more (FWHM $\left.\sim 100 \mathrm{~cm}^{-1}\right)$ increases in intensity and while the $\mathrm{G}$ band broadens and does not display well-resolved $\mathrm{G}^{-}$component any more. 


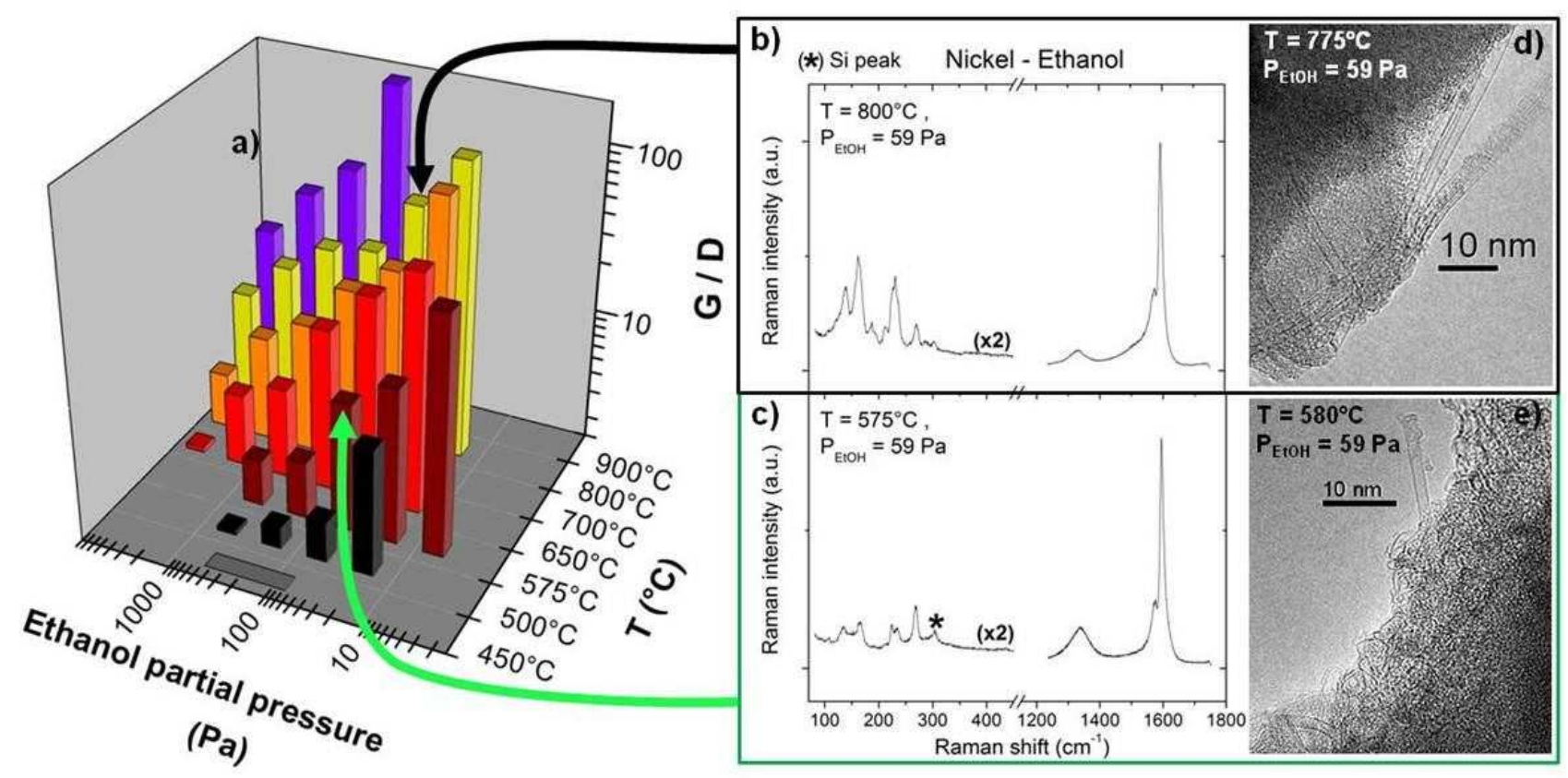

Figure 3. SWCNT samples grown with the nickel-ethanol couple: a) evolution of the G/D ratio as a function of temperature (T) and ethanol partial pressure, b-c) typical Raman spectra and d-e) HRTEM micrographs of samples prepared at high and low temperatures, respectively (see arrows). Note that pieces of amorphous $\mathrm{SiO}_{2}$ support are also visible in the micrographs. Only the four points in the high T-high P corner of fig. 3 a display the behavior shown in fig. 1 ; for those, fig. 3 a plots the maximum value of $I_{G} / I_{D}$ measured during the growth.

Samples grown in the high- and low-temperature regimes were characterized by HRTEM (Fig. 3d-e and Fig. S3 in Supporting Information). From HRTEM observations, high-temperature samples essentially display long bundles of SWCNT (>200 nm) with negligible deposits of amorphous carbon on their walls. No DWCNT or MWCNT could be observed. The SWCNT grown at high temperatures appear very straight with few defects. By contrast, low-temperature samples display defective and extremely short nanotubes. Some SWCNTs can reach $30 \mathrm{~nm}$ in length but most of them are less than 10nm long. SWCNT bundles are very scarce probably because there are too few long SWCNTs. Instead, the samples are dominated by agglomerates of very short and defective single-layer nanotubes mixed with catalyst nanoparticles. Their walls display several kinks but are essentially free of amorphous carbon 
deposits. Some structures are so short and defective that they may not be qualified of tubes anymore but of nanohorns or nanobubbles instead. However, their single-layer structure supports a certain degree of order. They are frequently observed to be connected to catalyst particles attesting of their catalytic formation.

Figure 4 shows the influence of the growth temperature and precursor pressure on the Raman features of samples grown with the nickel-ethanol system. The influence of the precursor pressure $(P)$ on the $I_{G} / I_{D}$ ratio is shown in figure $4 \mathrm{a}$ using a logarithmic scale for both axes. Contrary to varying the temperature, varying the precursor pressure has the advantage of only influencing the precursor supply and not all the other thermally-activated processes (decomposition, surface diffusion $\square$ ). The $\mathrm{I}_{\mathrm{G}} / \mathrm{I}_{\mathrm{D}}$ ratio follows a power law dependence as a function of the precursor pressure both low $\left(575^{\circ} \mathrm{C}\right)$ and high $\left(800^{\circ} \mathrm{C}\right)$ temperatures but with different slope values: -0.54 and -0.32 , respectively. Figure $4 b$ shows the temperature dependence of the $I_{G} / I_{D}$ ratio using an Arrhenius-type plot, i.e. $\ln \left(I_{G} / I_{D}\right)=f(1 / T)$. The plot reveals that $\mathrm{I}_{\mathrm{G}} / \mathrm{I}_{\mathrm{D}}$ increases with increasing temperature but according to two different Arrhenius-type laws at high and low temperatures. The associated activation energies are $0.3 \mathrm{eV}$ and $1.3 \mathrm{eV}$ above and below $680^{\circ} \mathrm{C}$, respectively. The two regimes are also clearly observed in the temperature dependence of the frequency and linewidth of the D band (fig. $4 \mathrm{c}, \mathrm{d}$ ). Above $680^{\circ} \mathrm{C}$, the frequency and FWHM of the D band are constant and close to $1328 \mathrm{~cm}^{-1}$ and $44 \mathrm{~cm}^{-1}$, respectively (at $\mathrm{E}_{\text {laser }}=2.33 \mathrm{eV}$ ). By contrast, under $680^{\circ} \mathrm{C}$, both the frequency and FWHM of the D band increase with decreasing temperature. All the catalytic systems that were investigated displayed these two distinct regimes in the temperature dependence of $\mathrm{I}_{\mathrm{G}} / \mathrm{I}_{\mathrm{D}}, \omega_{\mathrm{D}}$ and D-FWHM (see fig. S2). The apparent activation energies of $\mathrm{I}_{\mathrm{G}} / \mathrm{I}_{\mathrm{D}}$ measured for the different systems are in the range of $0.1-0.7 \mathrm{eV}$ at high temperature and of $1.3-3.5 \mathrm{eV}$ at low temperature (see table 1). Evidence of the existence of these two regimes can also be found in the literature. For instance, plotting the data of Kwok et al. [15] in an Arrhenius plot (see figure S4 in Supp. Info.) reveals two distinct regimes with activation energies of $0.1 \mathrm{eV}$ and $1.3 \mathrm{eV}$ at high and low temperatures, respectively. Studying samples grown with the ethanol-cobalt couple, Vinten et al. [14] 
also identified a change of regime in the temperature dependence of $I_{G} / I_{D}$ with an activation energy of 1.1 $\mathrm{eV}$ at low temperature, comparable to the value that we measured.
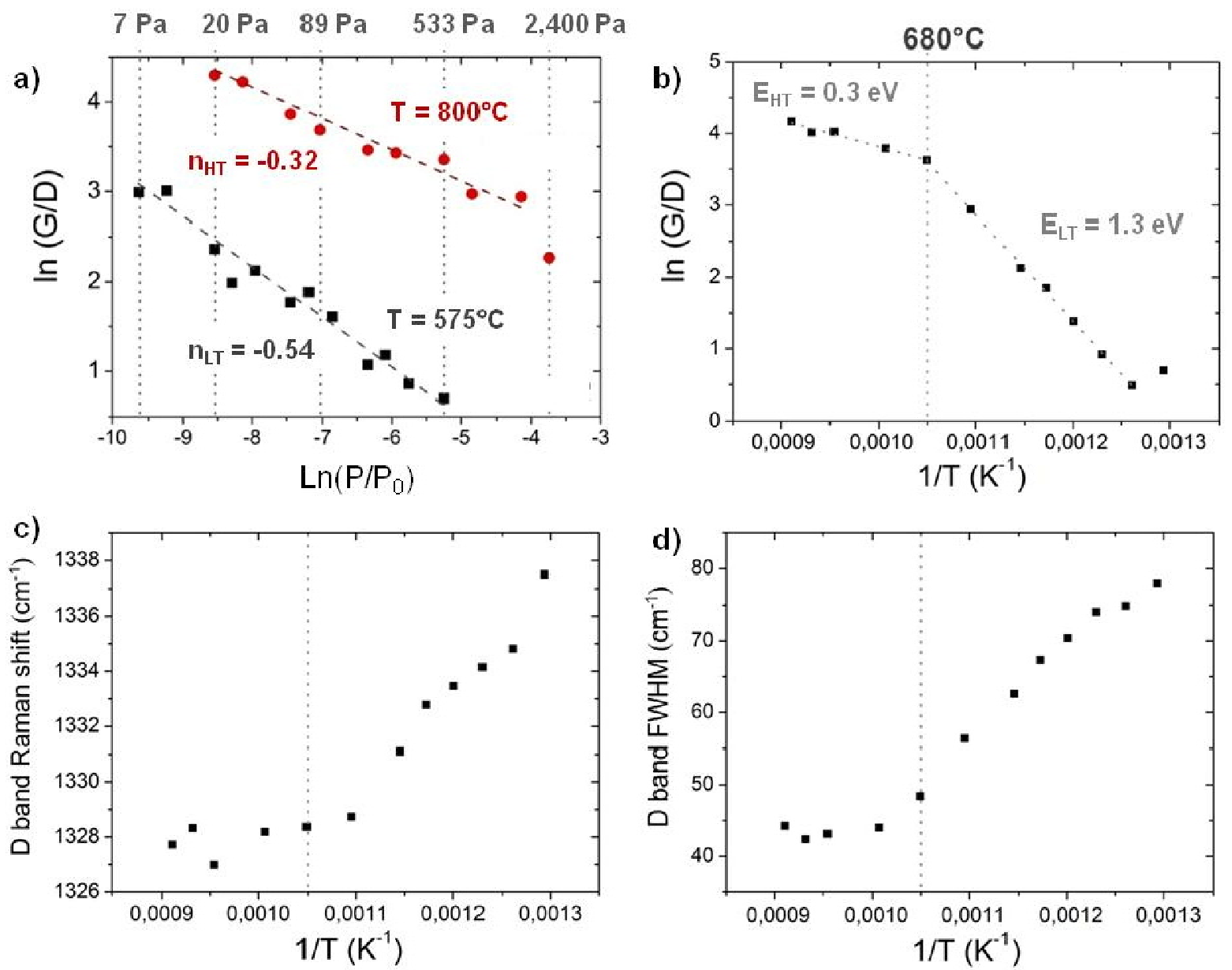

Figure 4. SWCNT samples grown with the nickel-ethanol couple: a) precursor pressure dependence of the $G / D$ ratio at $575^{\circ} \mathrm{C}$ and $800^{\circ} \mathrm{C}$, b) Arrhenius plot of the $\mathrm{G} / \mathrm{D}$ ratio at $P_{E t O H}=59 \mathrm{~Pa}, \mathrm{c}$ ) evolution of the frequency and $d$ ) of the linewidth of the D band. The laser energy is $E_{\text {laser }}=2.33 \mathrm{eV}$. The dashed lines in fig. 4a-b are fitted curves based on models $A$ and $B$.

Additional measurements were performed to assign the two components of the D band. First, we measured a reference sample of raw HiPCO SWCNT with a diameter distribution ranging from 0.7 to 1.3 $\mathrm{nm}$. The FWHM of the D band was $45 \mathrm{~cm}^{-1}$ and its position was $1332 \mathrm{~cm}^{-1}\left(\mathrm{E}_{\text {laser }}=2.33 \mathrm{eV}\right)$. From Gao et 
al. [19], an individual SWCNT has a D-band FWHM of about $20 \mathrm{~cm}^{-1}$ and its frequency can shift by about $30 \mathrm{~cm}^{-1}$ depending on its diameter (see also [26]). From the literature data, no broadening of the D band is expected with increasing the defect density as long as $\mathrm{L}_{\mathrm{D}}>5 \mathrm{~nm}$, which would correspond to a $\mathrm{I}_{\mathrm{G}} / \mathrm{I}_{\mathrm{D}}$ ratio of $\sim 0.3$ [34-36]. From these data, it can be inferred that the D-band FWHM of a standard SWCNT sample should be less than $50 \mathrm{~cm}^{-1}$. In contrast, a sample of pyrolytic carbon measured in the same experimental conditions displayed a FWHM of $120 \mathrm{~cm}^{-1}$. Second, two samples were subjected to oxidizing treatments under oxygen: i) a sample grown at low-temperature whose $\mathrm{D}$ band is dominated by the $D_{2}$ component and ii) a sample grown at high temperature whose $\mathrm{D}$ band is dominated by the $\mathrm{D}_{1}$ component. For both samples, the $\mathrm{D}_{1}$ component becomes more dominant with increasing oxidation time (see figure $\mathrm{S} 6$ ). The higher reactivity of the $\mathrm{D}_{2}$-related carbon structures to oxygen suggests a higher density of defects. After the oxidative treatment, the characteristic Raman features of SWCNTs (RBM, G-) were still observed without significant modification of the mode profiles.

From these data, the low-frequency $\mathrm{D}_{1}$ component is assigned to long SWCNTs with few defects (i.e. $\mathrm{L}_{\mathrm{D}}$ larger than 4-5 nm). The low frequency of $\mathrm{D}_{1}$ is attributed to the diameter effect specific of SWNTs [18] (see Table S5 in Supplementary Information for a comparison of different $\mathrm{sp}^{2}$ carbon materials). This attribution rationalizes why $\mathrm{D}_{1}$ and $\mathrm{D}_{2}$ are best resolved for the samples displaying the smallest diameter SWCNTs (i.e. cobalt-acetylene samples). It also agrees with the HRTEM observations that hightemperature samples consists of long and relatively defect-free SWCNTs.

Based on HRTEM observations, the broader and higher-frequency $\mathrm{D}_{2}$ band is assigned to very short and defective SWCNTs that dominate at low temperature. A higher density of defects is supported by the higher reactivity of the $\mathrm{D}_{2}$-related structures to oxidation. The upshift of the $\mathrm{D}_{2}$ component cannot be attributed to large diameter SWCNTs because it would disagree with the evolution of the SWCNT diameters from RBM profiles (see figure 2 and [30]). From the literature on graphene, a broadening of the $\mathrm{D}$ band without any significant shift is observed when $\mathrm{L}_{\mathrm{D}}$ decreases down to a few nanometers [17]. 
These D-band characteristics of highly defective graphene sheets are in good agreement with the observed features of the $\mathrm{D}_{2}$ component.

\section{Modeling}

From these observations, long and few-defects SWCNTs are preferentially grown at high temperature while catalyst particles produce short and defective single-walled carbon nanotubes and nanostructures at low temperature. This is a standard observation in non-catalytic thin film deposition techniques (MBE, PVD, CVD $\square$ ) that the crystalline quality of the film decreases with decreasing deposition temperature and increasing precursor supply [37,38]. This behavior is usually attributed to the kinetic competition between the supply of the gaseous precursor and the rearrangement of the surface intermediates. Experimentally, the apparent reaction orders $n_{H T}$ and $n_{L T}$ are negative, which means that increasing the supply of carbon increases the density of defects. Conceptually, this may be explained by considering that an excessive supply of carbon atoms tends to freeze the intermediate carbon structures, a phenomenon that is commonly observed during growth simulations $[5,6]$. In other words, the precursor pressure dependence may be explained by an inappropriate balance between the characteristic times of carbon supply and of annealing of the intermediate defective structures. However, this explanation alone cannot account for the temperature transition of the Arrhenius plot and for its convex shape. Convex Arrhenius plots usually put severe constraints on the possible microscopic models [29]. Hereafter are proposed two possible models that do reproduce the parameter dependence of the $I_{G} / I_{D}$ ratio.

In these models (figure 5), the molecules of gaseous carbon precursor (P) decompose at the surface of the catalyst particle to form reactive surface carbon species (C). Surface mobility allows those to create intermediate carbon structures at the nanotube rim. Model A (figure 5a) considers two possible intermediate states in rapid equilibrium: $I_{L T}$ and $I_{H T}$ dominant at low and high temperatures, respectively. For example, the intermediate states may differ by the configuration of the carbon atoms (e.g. pentagons, hexagons, heptagons, carbon chains $\square$ ) or by the state of the catalyst (solid/liquid, metal/carbide $\square$ ) as discussed later. By addition of carbon atoms, both intermediate states can be converted into either a $\mathrm{sp}^{2}$ 
hexagonal ring or a defective ring integrated in the nanotube wall. Model B (figure 5b) considers only one intermediate state. As in model A, this intermediate state can be converted and integrated in the nanotube as either a $\mathrm{sp}^{2}$ hexagonal ring or a defect. However, model $\mathrm{B}$ considers two possible mechanisms of defect creation: a low-activation-energy path dominant at low temperature and a highactivation-energy path dominant at high temperature. The nature of those mechanisms is left open by the kinetic model. As an example, the low-activation-energy path may correspond to the creation of defects promoted by carbon atoms only while the high-activation-energy path may correspond to the creation of defects assisted by reactive foreign species like water or oxygen.

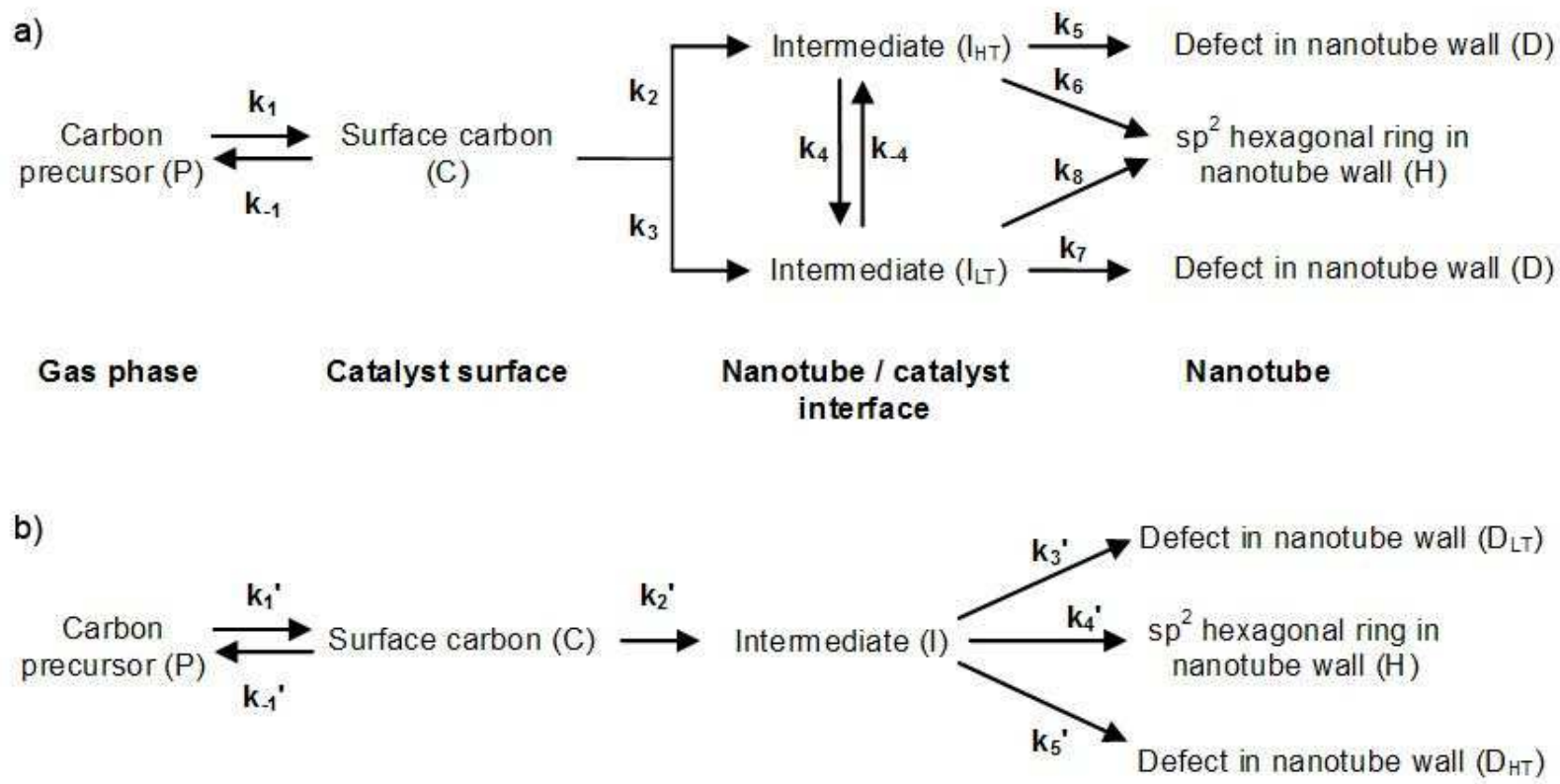

Figure 5. a) Model of defect integration with two intermediate states in equilibrium (model A). b) Model with two mechanisms of defect integration (model B).

Both models assume that, once the defects are fully integrated into the nanotube, their annealing is negligible at the considered growth temperatures. An additional step of deactivation could be added as for instance, in the model developed by Puretzky et al. [39]. However, this improvement would not modify the defect densities predicted by the models. To keep the models simple, the growth deactivation is therefore not considered here. 
We now focus on the concentration ratio $H / D$ of hexagonal rings to defects in the steady state regime and the resulting evolution of $\mathrm{I}_{\mathrm{G}} / \mathrm{I}_{\mathrm{D}}$ predicted by the models. As detailed in Supplementary Information, model A yields in the high temperature case:

$$
\ln \left(\frac{I_{G}}{I_{D}}\right)=A_{H T}-\frac{E_{H T}}{k T}+n_{H T} \ln \frac{P}{P_{0}}
$$

where $A_{H T}=\ln \left(K \frac{k_{6}^{0}}{k_{5}^{0}}\right), E_{H T}=E_{6}-E_{5}+n_{H T} \Delta_{r} G_{1}, n_{H T}=\frac{n_{6}-n_{5}}{n_{1}}$ and $\Delta_{r} G_{1}$ the standard Gibbs energy of dissociation of the precursor. $k_{x}=k_{x}^{0} \exp \left(-E_{x} / k T\right)$ is the kinetic constant of the elementary step $x$ with $k_{x}^{0}$ and $E_{x}$ the corresponding pre-exponential factor and activation energy, respectively; $n_{x}$ is the reaction order associated to surface carbon for the elementary step $x$.

In the low temperature case, model A brings:

$$
\ln \left(\frac{I_{G}}{I_{D}}\right)=A_{L T}-\frac{E_{L T}}{k T}+n_{L T} \ln \frac{P}{P_{0}}
$$

with $A_{L T}=\ln \left(K \frac{k_{8}^{0}}{k_{7}^{0}}\right), E_{L T}=E_{8}-E_{7}+n_{L T} \Delta_{r} G_{1}$ and $n_{L T}=\frac{n_{8}-n_{7}}{n_{1}}$.

Relations (1) and (2) both display Arrhenius-like dependences with respect to temperature and power law dependences with respect to the precursor pressure. The apparent pre-exponential factors, activation energies and reaction orders are different between high and low temperatures due to the change of reaction intermediates. Since the model puts no constraint on the relative order of $E_{L T}$ and $E_{H T}$, a convex Arrhenius plot is allowed.

Using similar assumptions, model B (figure 5b) yields in the high temperature case:

$$
\ln \left(\frac{I_{G}}{I_{D}}\right)=A_{H T}^{\prime}-\frac{E_{H T}^{\prime}}{k T}+n_{H T}^{\prime} \ln \frac{P}{P_{0}}
$$

where $A_{H T}^{\prime}=\ln \left(K \frac{k_{4}^{0 \prime}}{k_{5}^{0 \prime}}\right) ; \quad E_{H T}^{\prime}=E_{4}{ }^{\prime}-E_{5}{ }^{\prime}+n_{H T}{ }^{\prime} \Delta_{r} G_{1}$ and $n_{H T}{ }_{H T}=\frac{n_{4}{ }^{\prime}-n_{5}{ }^{\prime}}{n_{1}{ }^{\prime}} . \quad k_{x}{ }^{\prime}=k_{x}^{0{ }^{\prime}} \exp \left(-E_{x}{ }^{\prime} / k T\right)$ is the kinetic constant of the elementary step $x \square$ with $k_{x}^{0 \prime}$ and $E_{x}{ }^{\prime}$ the corresponding pre-exponential factor and 
activation energy, respectively; $n_{x} \square$ is the reaction order associated to surface carbon for the elementary step $x \square$

In the low temperature case, model B leads to:

$$
\ln \left(\frac{I_{G}}{I_{D}}\right)=A_{L T}^{\prime}-\frac{E_{L T}^{\prime}}{k T}+n_{L T}^{\prime} \ln \frac{P}{P_{0}}
$$

with $A_{L T}^{\prime}=\ln \left(K \frac{k_{4}^{0 \prime}}{k_{3}^{0 \prime}}\right), E_{L T}^{\prime}=E_{4}^{\prime}-E_{3}{ }^{\prime}+n_{L T}^{\prime} \Delta_{r} G_{1}$ and $n_{L T}^{\prime}=\frac{n_{4}{ }^{\prime}-n_{3}{ }^{\prime}}{n_{1}{ }^{\prime}}$.

As in model A, relations (3) and (4) both display Arrhenius-like dependences with respect to temperature and power law dependences with respect to the precursor pressure. In the case of model B, the change of apparent pre-exponential factors, activation energies and reaction orders between low and high temperature originates from two different mechanisms of defect creation. Since the model is based on $E_{3} \llbracket<E_{5}$, it follows that $E \sharp_{H T}<E \rrbracket_{{ }_{T}}$ and that model B corresponds to a convex Arrhenius plot.

The expressions of the parameter dependence of $I_{G} / I_{D}$ obtained from model A an B are mathematically equivalent. Using these relations to fit the experimental dependence of $I_{G} / I_{D}$ for the different catalystprecursor couples (fig. 2b, 4a-b, S2, S4) provides an excellent agreement. The obtained parameter values are summarized in Tables 1 and 2.

\begin{tabular}{lccccccc}
\hline Couple & $\mathbf{E}_{\mathbf{H T}}(\mathbf{e V})$ & $\mathbf{E}_{\mathrm{LT}}(\mathbf{e V})$ & $\mathbf{n}_{\mathrm{HT}}$ & $\mathbf{n}_{\mathrm{LT}}$ & $\mathbf{A}_{\mathrm{HT}}$ & $\mathbf{A}_{\mathrm{LT}}$ & Threshold temperature $\left({ }^{\circ} \mathbf{C}\right)$ \\
\hline Ni $\square$ ethanol & $0.32(0.06)$ & $1.3(0.1)$ & $-0.32(0.02)$ & $-0.54(0.03)$ & $5.0(0.7)$ & $16(1)$ & 680 \\
\hline
\end{tabular}

Table 1. Fit parameters of the temperature and precursor pressure dependences of $I_{G} / I_{D}$ for the nickel-ethanol couple.

\begin{tabular}{llll}
\hline $\mathbf{C o u p l e s}$ & $\mathbf{E}_{\mathbf{H T}}(\mathbf{e V})$ & $\mathbf{E}_{\mathbf{L T}}(\mathbf{e V})$ & Threshold temperature $\left({ }^{\circ} \mathbf{C}\right)$ \\
\hline $\mathbf{N i} \square$ methane & $0.7+/-0.1$ & $1.5+/-0.4$ & 625 \\
$\mathbf{N i} \square$ ethylene & $0.3+/-0.1$ & $1.4+/-0.1$ & 625 \\
$\mathbf{N i} \square$ acetylene & $0.4+/-0.1$ & $2.3+/-0.1$ & 600 \\
$\mathbf{C o} \square$ ethanol & $0.4+/-0.1$ & $1.1+/-0.1$ & 700 \\
$\mathbf{C o} \square$ acetylene & $0.1+/-0.1$ & $3.5+/-0.1$ & 700 \\
\hline
\end{tabular}

Table 2. Apparent activation energies at high growth temperatures $\left(E_{H T}\right)$ and low growth temperatures $\left(E_{L T}\right)$ for different carbon precursor-catalyst couples. 


\section{Discussion}

Both kinetic models are found to reproduce the experimental dependence of the density of defects. The modeling work significantly reduces the range of possible mechanisms but does not yet allow us to determine which one is the actual mechanism. Some suggestions and comments can still be made.

From model A, a first possibility is that the change of reaction path results from a phase transition of the catalyst. The solid or liquid state of the catalyst particle during nanotube CVD growth is still a debated question. Due to the Gibbs $\square$ Thomson effect [40], the melting temperature of catalyst nanoparticles of $1-2 \mathrm{~nm}$ can be depressed by up to $700-800^{\circ} \mathrm{C}$ as confirmed by calorimetry measurements [41]. Carbon uptake can further depress the melting temperature of the nanoparticles [42]. For low temperatures $\left(\right.$ e.g. $\left.480^{\circ} \mathrm{C}\right)$, recent in-situ transmission electron microscopy studies show the presence of lattice planes in the catalyst nanoparticles supporting that the catalyst particle was in the solid state $[43,44]$. It is therefore reasonable to assume that the melting temperature of the catalyst nanoparticles lies in the studied range of temperatures $\left(600-900^{\circ} \mathrm{C}\right)$. Experimentally, we observed threshold temperatures in the range of $600-680^{\circ} \mathrm{C}$ for nickel catalyst and in the range of $700-725^{\circ} \mathrm{C}$ for cobalt catalyst.

From model A, another possibility is a change of the carbon intermediates involved in the formation of the hexagonal network. Different types of carbon intermediates have already been considered such as adatoms, dimers, linear chains, pentagons, hexagons or heptagons. The conversion of carbon intermediates is frequently observed during growth simulations. For instance, Ohta et al. reported that short carbon chains frequently lead to the intermediate creation of pentagons and heptagons [45]. Pentagon-to-hexagon conversion was also observed to take place with the help of short-lived linear chains [46]. Interestingly, the nature of the dominant intermediates was observed to depend on the growth temperature [45].

From model B, a third possibility is a thermally-activated mechanism of defect creation. In presence of carbon and catalyst only, simulations show that defects tend to be thermally annealed [47]. However, the 
presence of additional compounds (water, oxygen, hydrogen $\square$ ) present as additives, by-products or contaminants may promote the creation of defects at high-temperature. For instance, nitrogensubstitution is observed when growing graphene or carbon nanotubes in presence of $\mathrm{NH}_{3}$ [48] or $\mathrm{N}_{2}$ [49]. Studying the growth temperature dependence of $I_{G} / I_{D}$ at controlled and varied amounts of additives would help assessing this hypothesis.

\section{Conclusion}

We reported on the evolution of the defect density of SWCNT grown by CCVD by measuring the $I_{G} / I_{D}$ ratio of samples grown in a large range of temperature and precursor pressure and from different catalyst-precursor couples. In situ Raman monitoring shows that the intrinsic $I_{G} / I_{D}$ ratio of as-grown SWCNTs can be masked by the deposition of amorphous carbon or modified by exposure to air. Concerning the influence of the catalyst nature on the defect density of nanotubes, nickel was systematically found to favor a lower density of defects than cobalt in similar experimental conditions. This is in agreement with the higher carbon affinity of cobalt that favors both a more rapid dissociation of the carbon precursor molecules and a lower surface mobility [50]. Concerning the influence of the carbon precursor, the defect density mainly depends on the reactivity of the precursor and on the partial pressure at which it is used. It is difficult to determine from our data the extent to which the precursor composition (e.g. oxygen content) also influences the defect density. In experimental conditions preventing these phenomena, the temperature dependence of the $I_{G} / I_{D}$ ratio systematically displays a convex Arrhenius plot, i.e. two different Arrhenius regimes with a lower activation energy at high temperature. The change of regime is also apparent in the characteristic features (frequency and linewidth) of the $\mathrm{D}$ band. The precursor pressure dependence of $I_{G} / I_{D}$ displays a power law whose reaction order decreases with increasing temperature. These results are correlated with HRTEM observations showing that long SWCNTs with few defects are grown in the high temperature regime while very short and defective SWCNTs are grown at low temperature. The convex Arrhenius behavior is well accounted by two different kinetic models: i) a model considering a change of intermediate states 
as a function of the temperature (for instance due to a phase transition of the catalyst particle or a change of carbon intermediates), and ii) a model considering the activation of second process of defect creation at high temperature (for instance due to the reaction with reactive gas species). Generally speaking, these findings support that disordered structures at the nanotube rim are important intermediates of nanotube growth that should be explicitly considered in atomic-scale growth models. 


\section{ACKNOWLEDGMENTS}

The authors acknowledge financial support from the Agence Nationale de la Recherche (P3N project SOS Nanotubes $\square$ ANR-09-NANO-028). The authors thank Walter Kob for fruitful theoretical discussions and Viviane Muffato, Saïd Tahir, David Maurin, Frédéric Pichot and Eric Alibert for technical assistance.

\section{SUPPORTING INFORMATION AVAILABLE}

Complementary Raman, HRTEM data, Arrhenius plots for all catalyst-precursor couples studied, detailed demonstrations for models A and B. 


\section{FIGURE CAPTIONS}

Figure 1. Time evolutions of the intensities of $G, D$ and of the G/D ratio (nickel-ethanol couple): a) standard behavior, b) high temperature/high pressure behavior. c) Evolution of the Raman spectra of SWCNT samples: as-grown (grey line), after $14 \mathrm{~h}$ of exposition to air (green line) and after annealing under Ar at $650^{\circ} \mathrm{C}$ (black line). The laser excitation line is $647 \mathrm{~nm}$.

Figure 2. SWCNT samples grown with the cobalt-acetylene couple: a) room-temperature Raman spectra as a function of the growth temperature, b) G/D ratio (diamonds) and proportion of the high frequency component (D2) in the D band (triangles), c) positions and d) FWHM of the two components of the D band against inverse temperature. The laser excitation line is $647 \mathrm{~nm}$. The dashed lines in fig. $2 b$ are fitted curves based on models $A$ and $B$.

Figure 3. SWCNT samples grown with the nickel-ethanol couple: a) evolution of the G/D ratio as a function of temperature (T) and ethanol partial pressure, $b$-c) typical Raman spectra and $d-e$ ) HRTEM micrographs of samples prepared at high and low temperatures, respectively (see arrows). Note that pieces of amorphous $\mathrm{SiO}_{2}$ support are also visible in the micrographs. Only the four points in the high T-high P corner of fig. 3 a display the behavior shown in fig. $1 b$; for those, fig. $3 a$ plots the maximum value of $I_{G} / I_{D}$ measured during the growth.

Figure 4. SWCNT samples grown with the nickel-ethanol couple: a) precursor pressure dependence of the $\mathrm{G} / \mathrm{D}$ ratio at $575^{\circ} \mathrm{C}$ and $800^{\circ} \mathrm{C}$, b) Arrhenius plot of the $\mathrm{G} / \mathrm{D}$ ratio at $P_{E t O H}=59 \mathrm{~Pa}, \mathrm{c}$ ) evolution of the frequency and d) of the linewidth of the D band. The laser energy is $E_{\text {laser }}=2.33 \mathrm{eV}$. The dashed lines in fig. 4a-b are fitted curves based on models $A$ and $B$.

Figure 5. a) Model of defect integration with two intermediate states in equilibrium. b) Model with two mechanisms of defect integration. 


\section{TABLES.}

Table 1. Fit parameters of the temperature and precursor pressure dependences of $I_{G} / I_{D}$ for the nickelethanol couple.

Table 2. Apparent activation energies at high growth temperatures $\left(E_{H T}\right)$ and low growth temperatures $\left(E_{L T}\right)$ for different carbon precursor-catalyst couples. 


\section{REFERENCES:}

1. Suzuki S, Kobayashi Y. Conductivity decrease in carbon nanotubes caused by lowacceleration-voltage electron irradiation. Jpn J Appl Phys 2005;44:1498-501.

2. Zhou Z, Jin R, Eres G, Subedi A, Mandrus D. Control of electron transport related defects in in situ fabricated single wall carbon nanotube devices. Appl Phys Lett 2006;89:133124-7.

3. Lee S, Kim G, Kim H, Choi BY, Lee J, Jeong BW, et al. Paired gap states in a semiconducting carbon nanotube: deep and shallow levels. Phys Rev Lett 2005;95:166402-6.

4. Dunlap BI. Relating carbon tubules. Phys Rev B 1994;49(8):5643.

5. Page AJ, Ohta Y, Irle S, Mokomura K. Mechanisms of single-walled carbon nanotube nucleation, growth, and healing determined using QM/MD methods. Acc Chem Res 2010;43:1375-85.

6. Amara H, Bichara C, Ducastelle F. Understanding the nucleation mechanisms of carbon nanotubes in catalytic chemical vapor deposition. Phys Rev Lett 2008;100(5):56105.

7. Wang X, Li Q, Xie J, Jin Z, Wang J, Li Y, et al. Fabrication of ultralong and electrically uniform single-walled carbon nanotubes on clean substrates. Nano Lett 2009;9(9):3137-41.

8. Dresselhaus MS, Jorio A, Souza Filho AG, Saito R. Defect characterization in graphene and carbon nanotubes using raman spectroscopy. Philos T Roy Soc A 2010;368:5355-77.

9. Pimenta M, Dresselhaus G, Dresselhaus MS, Cancado L, Jorio A, Saito R. Studying disorder in graphite-based systems by Raman spectroscopy. Phys Chem Chem Phys 2007;9(11):1276-90.

10. Tuinstra F, Koenig J. Raman spectrum of graphite. J Chem Phys 1970;53:1126.

11. Tuinstra F, Koenig J. Characterization of graphite fiber surfaces with Raman spectroscopy. J Compos Mater 1970;4(4):492.

12. Dresselhaus MS, Dresselhaus G, Saito R, Jorio A. Raman spectroscopy of carbon nanotubes. Phys Rep 2005;409(2):47-99.

13. Reich S, Thomsen C, Maultzsch J. Carbon nanotubes: basic concepts and physical properties. Berlin: Wiley/VCH; 2004.

14. Vinten P, Lefebvre J, Finnie P. Kinetic critical temperature and optimized chemical vapor deposition growth of carbon nanotubes. Chem Phys Lett 2009;469(4-6):293-7.

15. Kwok C, Reizman BJ, Agnew DE, Sandhu GS, Weistroffer J, Strano MS, et al. Temperature and time dependence study of single-walled carbon nanotube growth by catalytic chemical vapor deposition. Carbon 2010;48(4):1279-88.

16. Yasuda S, Hiraoka T, Futaba DN, Yamada T, Yumura M, Hata K. Existence and kinetics of graphitic carbonaceous impurities in carbon nanotube forests to assess the absolute purity. Nano Lett 2009;9(2):769-73. 
17. Lucchese M, Stavale F, Ferreira E, Vilani C, Moutinho M, Capaz RB, et al. Quantifying ioninduced defects and Raman relaxation length in graphene. Carbon 2010;48(5):1592-7.

18. Souza Filho A, Jorio A, Samsonidze GG, Dresselhaus G, Pimenta M, Dresselhaus M, et al. Competing spring constant versus double resonance effects on the properties of dispersive modes in isolated single-wall carbon nanotubes. Phys Rev B 2003;67(3):035427-74.

19. Gao B, Zhang Y, Zhang J, Kong J, Liu Z. Systematic comparison of the raman spectra of metallic and semiconducting SWNTs. J Phys Chem C 2008;112(22):8319-23.

20. Jorio A, Fantini C, Dantas M, Pimenta M, Souza Filho A, Samsonidze GG, et al. Linewidth of the Raman features of individual single-wall carbon nanotubes. Phys Rev B 2002;66(11):115411-9.

21. Tan PH, Dimovski S, Gogotsi Y. Raman scattering of non $\square$ planar graphite: arched edges, polyhedral crystals, whiskers and cones. Philos T Roy Soc A 2004;362(1824):2289.

22. Xu Z, Chen L, Liu L, Wu X. Structural changes in multi-walled carbon nanotubes caused by [gamma]-ray irradiation. Carbon 2010;49:350-1.

23. Matthews M, Pimenta M, Dresselhaus G, Dresselhaus M, Endo M. Origin of dispersive effects of the Raman D band in carbon materials. Phys Rev B 1999;59(10):6585-8.

24. Pócsik I, Hundhausen M, Koós M, Ley L. Origin of the D peak in the raman spectrum of microcrystalline graphite. J Non-Cryst Solids 1998;227:1083-6.

25. Ferrari AC, Robertson J. Raman spectroscopy of amorphous, nanostructured, diamond $\square$ like carbon, and nanodiamond. Philos T Roy Soc A 2004;362(1824):2477-512.

26. Arepalli S, Nikolaev P, Gorelik O, Hadjiev VG, Holmes W, Files B, et al. Protocol for the characterization of single-wall carbon nanotube material quality. Carbon 2004;42(8-9):1783-91.

27. Feng X, Liu K, Xie X, Zhou R, Zhang L, Li Q, et al. Thermal analysis study of the growth kinetics of carbon nanotubes and epitaxial graphene layers on them. J Phys Chem $\mathrm{C}$ 2009;113(22):9623-31.

28. Picher M, Anglaret E, Arenal R, Jourdain V. Self-deactivation of single-walled carbon nanotube growth studied by in situ raman measurements. Nano Lett 2009;9(2):542-7.

29. Truhlar DG, Kohen A. Convex Arrhenius plots and their interpretation. P Natl Acad Sci USA 2001;98(3):848.

30. Picher M, Anglaret E, Arenal R, Jourdain V. Processes controlling the diameter distribution of single-walled carbon nanotubes during catalytic chemical vapor deposition. ACS Nano 2011;5:2118 25 .

31. Li-Pook-Than A, Lefebvre J, Finnie P. Phases of carbon nanotube growth and population evolution from in situ Raman spectroscopy during chemical vapor deposition. J Phys Chem C 2010;114(25):11018-25.

32. Azam MA, Mohamed MA, Shikoh E, Fujiwara A. Thermal degradation of single-walled carbon nanotubes during alcohol catalytic chemical vapor deposition process. Jpn J Appl Phys 2010;49(2):02BA4 
33. Shim M, Gaur A, Nguyen KT, Abdula D, Ozel T. Spectral diversity in Raman G-band modes of metallic carbon nanotubes within a single chirality. J Phys Chem C 2008;112(33):13017-23.

34. Hulman M, Skákalová V, Roth S, Kuzmany H. Raman spectroscopy of single-wall carbon nanotubes and graphite irradiated by rays. J Appl Phys 2005;98:024311-6.

35. Dillon A, Parilla P, Alleman J, Gennett T, Jones K, Heben M. Systematic inclusion of defects in pure carbon single-wall nanotubes and their effect on the Raman D-band. Chem Phys Lett 2005;401(46):522-8.

36. Suzuki S, Yamaya K, Homma Y, Kobayashi Y. Activation energy of healing of low-energy irradiation-induced defects in single-wall carbon nanotubes. Carbon 2010;48(11):3211-7.

37. Saito Y, Harima H, Kurimoto E, Yamaguchi T, Teraguchi N, Suzuki A, et al. Growth temperature dependence of indium nitride crystalline quality grown by RF MBE. Phys Stat Sol B 2002;234(3):796-800.

38. Golecki I, Reidinger F, Marti J. Single crystalline, epitaxial cubic SiC films grown on (100) Si at $750^{\circ} \mathrm{C}$ by chemical vapor deposition. Appl Phys Lett 1992;60(14):1703-5.

39. Puretzky A, Geohegan D, Jesse S, Ivanov I, Eres G. In situ measurements and modeling of carbon nanotube array growth kinetics during chemical vapor deposition. Appl Phys A - Mater 2005;81(2):223-40.

40. Buffat P, Borel J. Size effect on the melting temperature of gold particles. Phys Rev A 1976;13(6):2287.

41. Harutyunyan A, Tokune T, Mora E. Liquid as a required catalyst phase for carbon single-walled nanotube growth. Appl Phys Lett 2005;87:051919.

42. Ding F, Bolton K, Rosén A. Iron-carbide cluster thermal dynamics for catalyzed carbon nanotube growth. J Vac Sci Technol, A 2004;22:1471.

43. Hofmann S, Sharma R, Ducati C, Du G, Mattevi C, Cepek C, et al. In Situ observations of catalyst dynamics during surface-bound carbon nanotube nucleation. Nano Lett 2007;7(3):602-8.

44. Yoshida H, Takeda S, Uchiyama T, Kohno H, Homma Y. Atomic-scale in-situ observation of carbon nanotube growth from solid state iron carbide nanoparticles. Nano Lett 2008;8(7):2082-6.

45. Ohta Y, Okamoto Y, Irle S, Morokuma K. Temperature dependence of iron-catalyzed continued single-walled carbon nanotube growth rates: density functional tight-binding molecular dynamics simulations. J Phys Chem C 2008;113(1):159-69.

46. Ohta Y, Okamoto Y, Page AJ, Irle S, Morokuma K. Quantum chemical molecular dynamics simulation of single-walled carbon nanotube cap nucleation on an iron particle. ACS Nano 2009;3(11):3413-20.

47. Karoui S, Amara H, Bichara C, Ducastelle F. Nickel-assisted healing of defective graphene. ACS Nano 2010;4:6114 20. 
48. Meyer JC, Kurasch S, Park HJ, Skakalova V, Künzel D, Groß A, et al. Experimental analysis of charge redistribution due to chemical bonding by high-resolution transmission electron microscopy. Nat Mater 2011;10:209-15.

49. Yu H, Zhang Q, Wang Q, Ning G, Luo G, Wei F. Effect of the reaction atmosphere on the diameter of single-walled carbon nanotubes produced by chemical vapor deposition. Carbon 2006;44(9):1706-12.

50. Somorjai GA, Li Y. Introduction to surface chemistry and catalysis: Wiley; 2010. 